\title{
Lyapunov inequality for a class of fractional differential equations with Dirichlet boundary conditions
}

\author{
Yasong Chen ${ }^{\mathrm{a}}$, Yeong-Cheng Liou ${ }^{\mathrm{b}, \mathrm{c}, *}$, Ching-Hua Lo ${ }^{\mathrm{d}, *}$ \\ a Department of Mathematics, Tianjin Polytechnic University, Tianjin 300387, China. \\ ${ }^{b}$ Department of Healthcare Administration and Medical Informatics, Center for Big Data Analytics \& Intelligent Healthcare \\ and Research Center of Nonlinear Analysis and Optimization, Kaohsiung Medical University, Kaohsiung 807, Taiwan. \\ ${ }^{c}$ Department of Medical Research, Kaohsiung Medical University Hospital, Kaohsiung 807, Taiwan. \\ ${ }^{d}$ Department of Management, Yango University, Fujian 350015, China.
}

Communicated by Y. H. Yao

\begin{abstract}
In this paper we present Lyapunov inequality for the following fractional boundary value problem

$$
\left\{\begin{array}{l}
\frac{d}{d t}\left(\frac{1}{2}{ }_{a} D_{t}^{-\beta} u^{\prime}(t)+\frac{1}{2}{ }_{t} D_{b}^{-\beta} u^{\prime}(t)\right)+w(t) u(t)=0, \quad a<t<b, \\
u(a)=u(b)=0
\end{array}\right.
$$

where ${ }_{a} D_{t}^{-\beta}$ and ${ }_{t} D_{b}^{-\beta}$ are the left and right Riemann-Liouville fractional integrals of order $0 \leqslant \beta<1$, respectively, and $\omega \in \mathrm{L}^{1}([a, b], \mathbb{R})$. Using the obtained inequality, we provide lower bounds for the first eigenvalue of the fractional differential equations with homogeneous Dirichlet boundary problem. (C)2017 All rights reserved.
\end{abstract}

Keywords: Lyapunov type inequality, fractional differential equations, boundary value problem, eigenvalue. 2010 MSC: 15A42, 34A08, 35P15.

\section{Introduction}

The classical Lyapunov inequality states that, if $u$ is a nontrivial solution of the following linear boundary value problem

$$
\left\{\begin{array}{l}
u^{\prime \prime}(t)+w(t) u(t)=0, \quad a<t<b \\
u(a)=u(b)=0
\end{array}\right.
$$

where $\omega:[a, b] \rightarrow \mathbb{R}$ is a continuous function, then

$$
\int_{a}^{b}|\omega(t)| d t>\frac{4}{b-a} .
$$

\footnotetext{
*Corresponding authors

Email addresses: yasongchen@126.com (Yasong Chen), simplex_liou@hotmail.com (Yeong-Cheng Liou), bde_lo@sina.com (Ching-Hua Lo)

doi:10.22436/jnsa.010.06.44
}

Received 2017-05-01 
Inequality (1.2) has been proved to be very useful in various applications including oscillation theory, stability criteria for periodic differential equations, estimates for intervals of disconjugacy, and eigenvalue bounds for ordinary differential equations. Many improvements and generalizations have been obtained, meanwhile, different proofs of this inequality have appeared in the literature. We refer the reader to $[1,17]$ and the references therein.

For the nonlinear case, Elbert [5] firstly extended the inequality (1.2) to the following p-Laplacian problem

$$
\left\{\begin{array}{l}
\left(\left|u^{\prime}\right|^{p-2} u^{\prime}\right)^{\prime}+w(t)|u|^{p-2} u=0, \quad a<t<b \\
u(a)=u(b)=0
\end{array}\right.
$$

where $\omega \in \mathrm{L}^{1}([\mathrm{a}, \mathrm{b}], \mathbb{R})$ and $1<\mathrm{p}<\infty$, and obtained the following inequality

$$
\int_{a}^{b}|\omega(t)| d t>\frac{2^{p}}{(b-a)^{p-1}} .
$$

For $p=2$, then the linear problem (1.1) is recovered. Nápoli and Pinasco [4] extended the inequality (1.3) to the following more generalized nonlinear problems

$$
\left\{\begin{array}{l}
\left(\psi\left(u^{\prime}\right)\right)^{\prime}+w(t) \psi(u)=0, \quad a<t<b \\
u(a)=u(b)=0
\end{array}\right.
$$

where $\psi: \mathbb{R} \rightarrow \mathbb{R}$ is a convex nondecreasing function satisfying a certain condition. For a review of recent developments in these problems, we refer the reader to the book [15].

Instead of the (classical) ordinary derivative in (1.1), recently, many researchers have focused their attention on the Lyapunov-type inequalities for one-dimensional fractional boundary value problems. In this direction, Ferreira [6] for the first time established a fractional version of inequality (1.2) for the following boundary value problem

$$
\left\{\begin{array}{l}
\left({ }_{a} D^{\alpha} \mathfrak{u}\right)(t)+w(t) u(t)=0, \quad a<t<b, 1<\alpha \leqslant 2, \\
\mathfrak{u}(a)=u(b)=0
\end{array}\right.
$$

where ${ }_{a} \mathrm{D}^{\alpha}$ denotes the Riemann-Liouville fractional derivative of order $\alpha$. Explicitly, the author showed that if the above problem (1.4) has a nontrivial solution, then

$$
\int_{a}^{b}|\omega(t)| d t>\Gamma(\alpha)\left(\frac{4}{b-a}\right)^{\alpha-1}
$$

which yields the standard Lyapunov inequality (1.2) if we take $\alpha=2$ in (1.5), where $\Gamma$ is the gamma function. From then on, some Lyapunov-type inequalities for other fractional boundary value problems were established, see, for example, $[7,10,11,14,16]$ and the references listed therein. On the other hand, there are some papers on Lyapunov-type inequalities for partial differential equations, we refer the reader to $[2,3,9]$ for related results.

Motivated by the above works, in present paper, we focus our attention the following fractional boundary value problem

$$
\left\{\begin{array}{l}
\frac{d}{d t}\left(\frac{1}{2}{ }_{a} D_{t}^{-\beta} u^{\prime}(t)+\frac{1}{2}{ }_{t} D_{b}^{-\beta} u^{\prime}(t)\right)+w(t) u(t)=0, \quad a<t<b, \\
u(a)=u(b)=0,
\end{array}\right.
$$

where ${ }_{a} D_{t}^{-\beta}$ and ${ }_{t} D_{b}^{-\beta}$ are the left and right Riemann-Liouville fractional integrals of order $0 \leqslant \beta<$ 1 , respectively, and $\omega \in \mathrm{L}^{1}([a, b], \mathbb{R})$. To the best of our knowledge, there is no literature to discuss the Lyapunov type inequality for fractional differential equations with left and right Riemann-Liouville fractional integrals. The purpose of this paper is to address this issue for the BVP (1.6) and establish the following result. 
Theorem 1.1. Let $\omega \in \mathrm{L}^{1}([\mathrm{a}, \mathrm{b}], \mathbb{R})$ be nonnegative. Suppose that $B V P(1.6)$ has a nontrivial solution. Then

$$
\int_{a}^{b}|\omega(t)| d t \geqslant\left(\frac{2(b-a)^{\left(\alpha-\frac{1}{2}\right)}}{\Gamma(\alpha)(2 \alpha-1)^{\frac{1}{2}}} \frac{1}{|\cos (\pi \alpha)|^{\frac{1}{2}}}\right)^{-2} .
$$

The paper is organized as follows. In Section 2, we recall some basic concepts and properties of the Riemann-Liouville fractional integral and the Caputo fractional derivative of order $\alpha \geqslant 0$ which will be used further in this paper. In Section 3, we prove the Lyapunov-type inequality (1.7) for the BVP (1.6), and give the lower bound for the first eigenvalue of the homogeneous Dirichlet boundary value problem.

\section{Preliminary results}

In this section, for the reader's convenience, we collect some basic definitions and properties that will be used in the sequel, see [12].

Definition 2.1 (Left and Right Riemann-Liouville fractional integrals). Let $u$ be a function defined on $[a, b]$. The left and right Riemann-Liouville fractional integrals of order $\alpha>0$ for function $u$ denoted by ${ }_{a} D_{t}^{-\alpha} \mathfrak{u}(t)$ and ${ }_{t} D_{b}^{-\alpha} \mathfrak{u}(t)$, respectively, are defined by

$$
{ }_{a} D_{t}^{-\alpha} \mathfrak{u}(t)=\frac{1}{\Gamma(\alpha)} \int_{a}^{t}(t-s)^{\alpha-1} \mathfrak{u}(s) d s, \quad t \in[a, b],
$$

and

$$
{ }_{t} D_{b}^{-\alpha} u(t)=\frac{1}{\Gamma(\alpha)} \int_{t}^{b}(s-t)^{\alpha-1} u(s) d s, \quad t \in[a, b],
$$

provided the right-hand sides are pointwise defined on $[a, b]$, where $\Gamma>0$ is the gamma function.

Definition 2.2 (Left and Right Riemann-Liouville fractional derivatives). Let $u$ be a function defined on $[a, b]$. The left and right Riemann-Liouville fractional derivatives of order $\alpha>0$ for function $u$ denoted by ${ }_{a} D_{t}^{\alpha} \mathfrak{u}(t)$ and ${ }_{t} D_{b}^{\alpha} \mathfrak{u}(t)$, respectively, are defined by

$$
{ }_{a} D_{t}^{\alpha} u(t)=\frac{d^{n}}{d t^{n}} a_{t}^{\alpha-n} u(t)
$$

and

$$
{ }_{t} D_{b}^{\alpha} u(t)=(-1)^{n} \frac{d^{n}}{d t^{n}}{ }_{t} D_{b}^{\alpha-n} u(t)
$$

where $\mathrm{t} \in[\mathrm{a}, \mathrm{b}], \mathrm{n}-1 \leqslant \alpha<\mathrm{n}$ and $\mathrm{n} \in \mathbb{N}$.

The left and right Caputo fractional derivatives are defined via the above Riemann-Liouville fractional derivatives. In particular, they are defined for the function belonging to the space of absolutely continuous functions, which is denoted by $A C([a, b], \mathbb{R}) . A C^{k}([a, b], \mathbb{R})(k=1,2, \cdots)$ is the space of functions $u$ such that $u \in C^{k-1}([a, b], \mathbb{R})$ and $u^{(k-1)} \in A C([a, b], \mathbb{R})$. Especially, $A C([a, b], \mathbb{R})=A C^{1}([a, b], \mathbb{R})$.

Definition 2.3. Let $\alpha \geqslant 0$ and $n \in \mathbb{N}$. If $\alpha \in(n-1, n)$ and $u \in A C^{n}([a, b], \mathbb{R})$, then the left and right Caputo fractional derivatives of order $\alpha$ for function $u$ denoted by ${ }_{a}^{c} D_{t}^{\alpha} u(t)$ and ${ }_{t}^{c} D_{b}^{\alpha} u(t)$, respectively, exist almost everywhere on $[a, b] .{ }_{a}^{c} D_{t}^{\alpha} \mathfrak{u}(t)$ and ${ }_{t}^{c} D_{b}^{\alpha} \mathfrak{u}(t)$ are represented by

$$
{ }_{a}^{c} D_{t}^{\alpha} u(t)={ }_{a} D_{t}^{\alpha-n} u^{(n)}(t)=\frac{1}{\Gamma(n-\alpha)} \int_{a}^{t}(t-s)^{n-\alpha-1} u^{(n)}(s) d s,
$$

and

$$
{ }_{t}^{c} D_{b}^{\alpha} u(t)=(-1)^{n}{ }_{t} D_{b}^{\alpha-n} u^{(n)}(t)=\frac{(-1)^{n}}{\Gamma(n-\alpha)} \int_{t}^{b}(s-t)^{n-\alpha-1} u^{(n)}(s) d s,
$$


respectively, where $t \in[a, b]$. In particular, if $0<\alpha<1$, then

$$
{ }_{a}^{c} D_{t}^{\alpha} u(t)={ }_{a} D_{t}^{\alpha-1} u^{\prime}(t)=\frac{1}{\Gamma(1-\alpha)} \int_{a}^{t}(t-s)^{-\alpha} u^{\prime}(s) d s, \quad t \in[a, b],
$$

and

$$
{ }_{\mathrm{t}}^{\mathrm{c}} \mathrm{D}_{\mathrm{b}}^{\alpha} \mathrm{u}(\mathrm{t})=-{ }_{\mathrm{t}} \mathrm{D}_{\mathrm{b}}^{\alpha-1} \mathrm{u}^{\prime}(\mathrm{t})=\frac{-1}{\Gamma(1-\alpha)} \int_{\mathrm{t}}^{\mathrm{b}}(\mathrm{s}-\mathrm{t})^{-\alpha} \mathrm{u}^{\prime}(\mathrm{s}) \mathrm{ds}, \quad \mathrm{t} \in[\mathrm{a}, \mathrm{b}] .
$$

If $\alpha=n-1, u \in A C^{n-1}([a, b], \mathbb{R})$, then ${ }_{a}^{c} D_{t}^{n-1} u(t)$ and ${ }_{t}^{c} D_{b}^{n-1} u(t)$ are represented by

$$
{ }_{a}^{c} D_{t}^{n-1} u(t)=u^{(n-1)}(t) \quad \text { and } \quad{ }_{t}^{c} D_{b}^{n-1} u(t)=(-1)^{(n-1)} u^{(n-1)}(t), \quad t \in[a, b] .
$$

Based on the above definitions, we recall some of the properties of the Riemann-Liouville integral and derivative operators.

Property 2.4. The left and right Riemann-Liouville fractional integral operators have the property of a semigroup, that is, for all $\alpha_{1}, \alpha_{2}>0$,

$$
{ }_{a} D_{t}^{-\alpha_{1}}\left({ }_{a} D_{t}^{-\alpha_{2}} u(t)\right)={ }_{a} D_{t}^{-\alpha_{1}-\alpha_{2}} u(t)
$$

and

$$
{ }_{t} D_{b}^{-\alpha_{1}}\left({ }_{t} D_{b}^{-\alpha_{2}} u(t)\right)={ }_{t} D_{b}^{-\alpha_{1}-\alpha_{2}} u(t),
$$

in any point $\mathrm{t} \in[\mathrm{a}, \mathrm{b}]$ for continuous function $\mathrm{u}$ and for almost every point in $[\mathrm{a}, \mathrm{b}]$ if the function $\mathrm{u} \in \mathrm{L}^{1}([\mathrm{a}, \mathrm{b}], \mathbb{R})$.

Property 2.5. The left and right Riemann-Liouville fractional integral operators have the following property

$$
\int_{a}^{b}\left[{ }_{a} D^{-\gamma} f(t)\right] g(t) d t=\int_{a}^{b}\left[{ }_{t} D_{b}^{-\gamma} g(t)\right] f(t) d t, \quad \gamma>0,
$$

provided that $f \in \mathrm{L}^{\mathrm{p}}([\mathrm{a}, \mathrm{b}], \mathbb{R}), \mathrm{g} \in \mathrm{L}^{\mathrm{q}}([\mathrm{a}, \mathrm{b}], \mathbb{R})$ and $\mathrm{p} \geqslant 1, \mathrm{q} \geqslant 1, \frac{1}{\mathrm{p}}+\frac{1}{\mathrm{q}} \leqslant \frac{1}{\gamma}$ or $\mathrm{p} \neq 1, \mathrm{q} \neq 1, \frac{1}{\mathrm{p}}+\frac{1}{\mathrm{q}}=1+\gamma$.

The composition of the Riemann-Liouville fractional integration operator with the Caputo fractional differentiation operator is given by the following result.

Property 2.6. Let $\mathrm{n} \in \mathbb{N}$ and $\mathrm{n}-1<\gamma \leqslant \mathrm{n}$. If $u \in A C^{\mathfrak{n}}([\mathrm{a}, \mathrm{b}], \mathbb{R})$ or $\mathrm{u} \in \mathrm{C}^{\mathfrak{n}}([\mathrm{a}, \mathrm{b}], \mathbb{R})$, then

$$
{ }_{a} D_{t}^{-\gamma}\left({ }_{a}^{c} D_{t}^{\gamma} u(t)\right)=u(t)-\sum_{j=0}^{n-1} \frac{u^{(j)}(a)}{j !}(t-a)^{j},
$$

and

$$
{ }_{t} D_{b}^{-\gamma}\left({ }_{t}^{c} D_{b}^{\gamma} u(t)\right)=u(t)-\sum_{j=0}^{n-1} \frac{(-1)^{j} u^{(j)}(b)}{j !}(b-t)^{j},
$$

for $\mathrm{t} \in[\mathrm{a}, \mathrm{b}]$. In particular, if $0<\gamma \leqslant 1$, and $\mathrm{u} \in A \mathrm{C}([\mathrm{a}, \mathrm{b}], \mathbb{R})$ or $\mathrm{u} \in \mathrm{C}^{1}([\mathrm{a}, \mathrm{b}], \mathbb{R})$, then

$$
{ }_{a} D_{t}^{-\gamma}\left({ }_{a}^{c} D_{t}^{\gamma} u(t)\right)=u(t)-u(a) \text {, and }{ }_{t} D_{b}^{-\gamma}\left({ }_{t}^{c} D_{b}^{\gamma} u(t)\right)=u(t)-u(b) \text {. }
$$

Property 2.7. Let $\mathrm{n} \in \mathbb{N}$, and $\mathrm{n}-1<\gamma<\mathrm{n}$. If $\mathrm{f}$ is a function defined on $[\mathrm{a}, \mathrm{b}]$ for which the Caputo fractional derivatives ${ }_{\mathrm{a}}^{\mathrm{c}} \mathrm{D}_{\mathrm{t}}^{\gamma} \mathrm{f}(\mathrm{t})$ and ${ }_{\mathrm{t}}^{\mathrm{c}} \mathrm{D}_{\mathrm{b}}^{\gamma} \mathrm{f}(\mathrm{t})$ of order $\gamma$ exist together with the Riemann-Liouville fractional derivatives ${ }_{a} D_{t}^{\gamma} f(t)$ and ${ }_{t} D_{b}^{\gamma} f(t)$, then

$$
{ }_{a}^{c} D_{t}^{\gamma} f(t)={ }_{a} D_{t}^{\gamma} f(t)-\sum_{j=0}^{n-1} \frac{f^{(j)}(a)}{\Gamma(j-\gamma+1)}(t-a)^{j-\gamma}, \quad t \in[a, b],
$$


and

$$
{ }_{\mathrm{t}}^{\mathrm{c}} \mathrm{D}_{\mathrm{b}}^{\gamma} \mathrm{f}(\mathrm{t})={ }_{\mathrm{t}} \mathrm{D}_{\mathrm{b}}^{\gamma} \mathrm{f}(\mathrm{t})-\sum_{j=0}^{\mathrm{n}-1} \frac{f^{(j)}(\mathrm{b})}{\Gamma(j-\gamma+1)}(b-t)^{j-\gamma}, \quad t \in[a, b] .
$$

In particular, when $0<\gamma<1$, we have

$$
{ }_{a}^{c} D_{t}^{\gamma} f(t)={ }_{a} D_{t}^{\gamma} f(t)-\frac{f(a)}{\Gamma(1-\gamma)}(t-a), \quad t \in[a, b],
$$

and

$$
{ }_{t}^{c} D_{b}^{\gamma} f(t)={ }_{t} D_{b}^{\gamma} f(t)-\frac{f(b)}{\Gamma(1-\gamma)}(b-t), \quad t \in[a, b]
$$

\section{Proof of the main result}

The purpose of this section is to finish the proof of Theorem 1.1. For the convenience, we firstly recall some fractional spaces, see [8] for more details. To this end, denote by $L^{p}([a, b], \mathbb{R})(1<p<+\infty)$ the Banach space of functions on $[a, b]$ with values in $\mathbb{R}$ under the norm

$$
\|u\|_{p}=\left(\int_{a}^{b}|u(t)|^{p} d t\right)^{1 / p}
$$

and $L^{\infty}([a, b], \mathbb{R})$ is the Banach space of essentially bounded functions from $[a, b]$ into $\mathbb{R}$ equipped with the norm

$$
\|u\|_{\infty}=\operatorname{ess} \sup \{|u(t)|: t \in[a, b]\} .
$$

For $0<\alpha \leqslant 1$ and $1<p<+\infty$, the fractional derivative space $E_{0}^{\alpha, p}$ is defined by

$$
\begin{aligned}
\mathrm{E}_{0}^{\alpha, p} & =\left\{\mathrm{u} \in \mathrm{L}^{\mathrm{p}}([\mathrm{a}, \mathrm{b}], \mathbb{R}):{ }_{a} \mathrm{D}_{\mathrm{t}}^{\alpha} \mathrm{u} \in \mathrm{L}^{\mathrm{p}}([\mathrm{a}, \mathrm{b}], \mathbb{R}) \text { and } \mathrm{u}(\mathrm{a})=\mathrm{u}(\mathrm{b})=0\right\} \\
& =\overline{\mathrm{C}_{0}^{\infty}([\mathrm{a}, \mathrm{b}], \mathbb{R})} \|^{\|,\|_{\alpha, p}},
\end{aligned}
$$

where $\|\cdot\|_{\alpha, p}$ is defined as follows

$$
\|u\|_{\alpha, p}=\left(\int_{a}^{b}|u(t)|^{p} d t+\int_{a}^{b}\left|{ }_{a} D_{t}^{\alpha} u(t)\right|^{p} d t\right)^{1 / p} .
$$

Then $\mathrm{E}_{0}^{\alpha, p}$ is a reflexive and separable Banach space. Moreover, $\mathrm{E}_{0}^{\alpha, p} \in \mathrm{C}([\mathrm{a}, \mathrm{b}], \mathbb{R})$.

Lemma 3.1 ([8, Proposition 3.2]). Let $0<\alpha \leqslant 1$ and $1<p<+\infty$. For all $u \in E_{0}^{\alpha, p}$, if $\alpha>\frac{1}{p}$, we have

$$
\|u\|_{p} \leqslant \frac{(b-a)^{\alpha}}{\Gamma(\alpha+1)}\left\|_{0} D_{t}^{\alpha} u\right\|_{p}
$$

Remark 3.2. According to (3.1) and (3.2), we can consider the following norm in $\mathrm{E}_{0}^{\alpha, p}$

$$
\|u\|_{\alpha, p}=\left\|_{0} D_{t}^{\alpha} u\right\|_{p}
$$

which is equivalent to (3.1).

In what follows, we treat BVP (1.6) in the space $E_{0}^{\alpha, p}$ with $p=2$ and $\frac{1}{2}<\alpha \leqslant 1$ with $\alpha=1-\frac{\beta}{2}$. According to Remark 3.2, for any $u \in E_{0}^{\alpha, 2}$, the corresponding norm can be defined by $\|u\|_{\alpha, 2}=\left\|_{0} D_{t}^{\alpha} u\right\|_{2}$.

Lemma 3.3. For any $\mathrm{u} \in \mathrm{E}_{0}^{\alpha, 2}$, we have

$$
\left|u\left(t_{2}\right)-u\left(t_{1}\right)\right| \leqslant \frac{2\left(t_{2}-t_{1}\right)^{\left(\alpha-\frac{1}{2}\right)}}{\Gamma(\alpha)(2 \alpha-1)^{\frac{1}{2}}}\|u\|_{\alpha, 2}, \quad a \leqslant t_{1}<t_{2} \leqslant b .
$$


Proof. The proof can be found in [8, Proposition 3.3], so we omit its details.

Lemma 3.4 ([8, Proposition 4.1]). For any $\mathrm{u} \in \mathrm{E}_{0}^{\alpha, 2}$, we have

$$
|\cos (\pi \alpha)|\|u\|_{\alpha, 2}^{2} \leqslant-\int_{a}^{b}{ }_{a} D_{t}^{\alpha} u(t){ }_{t} D_{b}^{\alpha} u(t) d t \leqslant \frac{1}{|\cos (\pi \alpha)|}\|u\|_{\alpha, 2}^{2} .
$$

Now we are in the position to prove Theorem 1.1.

Proof. Let $u$ be a nontrivial solution of BVP (1.6). Then, multiplying by $u$ and integrating by parts, we obtain, in view of Properties 2.4 and 2.5, that

$$
\begin{aligned}
\int_{a}^{b} w(t) u^{2}(t) d t & =-\frac{1}{2} \int_{a}^{b} \frac{d}{d t}\left({ }_{a} D_{t}^{-\beta} u^{\prime}(t)+{ }_{t} D_{b}^{-\beta} u^{\prime}(t)\right) u(t) d t \\
& =\frac{1}{2} \int_{a}^{b}\left({ }_{a} D_{t}^{-\beta} u^{\prime}(t)+{ }_{t} D_{b}^{-\beta} u^{\prime}(t)\right) u^{\prime}(t) d t \\
& =\frac{1}{2} \int_{a}^{b}\left({ }_{a} D_{t}^{-\frac{\beta}{2}} u^{\prime}(t){ }_{t} D_{b}^{-\frac{\beta}{2}} u^{\prime}(t)+{ }_{t} D_{b}^{\frac{-\beta}{2}} u^{\prime}(t){ }_{a} D_{t}^{-\frac{\beta}{2}} u^{\prime}(t)\right) d t
\end{aligned}
$$

In addition, due to the fact that $u(a)=u(b)=0$ and on account of (2.1), (2.2), (2.3), (2.4), one deduces that

$$
{ }_{a} D_{t}^{-\frac{\beta}{2}} u^{\prime}(t)={ }_{a} D^{1-\frac{\beta}{2}} u(t) \text { and }{ }_{t} D_{b}^{-\frac{\beta}{2}} u^{\prime}(t)=-{ }_{t} D_{b}^{1-\frac{\beta}{2}} u(t)
$$

Substituting (3.4) into (3.3), we have

$$
\int_{a}^{b} w(t) u^{2}(t) d t=-\int_{a}^{b}{ }_{a} D^{1-\frac{\beta}{2}} u(t){ }_{t} D_{b}^{1-\frac{\beta}{2}} u(t) d t
$$

On the other hand, since $u(t)$ is continuous, let us choose $c \in[a, b]$ where $|u(t)|$ achieves its maximum. Then, according to Lemma 3.3, we conclude that, for $t_{1}=c$ and $t_{2}=b$,

$$
|u(c)| \leqslant \frac{2\left(t_{2}-c\right)^{\left(\alpha-\frac{1}{2}\right)}}{\Gamma(\alpha)(2 \alpha-1)^{\frac{1}{2}}}\|u\|_{\alpha, 2}
$$

which, combining with Lemma 3.4 and (3.5), yields that

$$
\begin{aligned}
|u(c)| & \leqslant \frac{2\left(t_{2}-c\right)^{\left(\alpha-\frac{1}{2}\right)}}{\Gamma(\alpha)(2 \alpha-1)^{\frac{1}{2}}} \frac{1}{|\cos (\pi \alpha)|^{\frac{1}{2}}}\left(-\int_{a}^{b} a D^{1-\frac{\beta}{2}} u(t)_{t} D_{b}^{1-\frac{\beta}{2}} u(t) d t\right)^{\frac{1}{2}} \\
& =\frac{2\left(t_{2}-c\right)^{\left(\alpha-\frac{1}{2}\right)}}{\Gamma(\alpha)(2 \alpha-1)^{\frac{1}{2}}} \frac{1}{|\cos (\pi \alpha)|^{\frac{1}{2}}}\left(\int_{a}^{b} \omega(t) u^{2}(t) d t\right)^{\frac{1}{2}} \\
& \leqslant \frac{2(b-a)^{\left(\alpha-\frac{1}{2}\right)}}{\Gamma(\alpha)(2 \alpha-1)^{\frac{1}{2}}} \frac{1}{|\cos (\pi \alpha)|^{\frac{1}{2}}}\left(\int_{a}^{b} \omega(t) u^{2}(t) d t\right)^{\frac{1}{2}} \\
& \leqslant \frac{2(b-a)^{\left(\alpha-\frac{1}{2}\right)}}{\Gamma(\alpha)(2 \alpha-1)^{\frac{1}{2}}} \frac{|u(c)|}{|\cos (\pi \alpha)|^{\frac{1}{2}}}\left(\int_{a}^{b} \omega(t) d t\right)^{\frac{1}{2}} .
\end{aligned}
$$

Therefore, the conclusion is proved.

In what follows, using the above obtained inequality (1.7), we give lower bounds for the first eigenvalue of the following eigenvalue problem

$$
\left\{\begin{array}{l}
\frac{d}{d t}\left(\frac{1}{2}{ }_{a} D_{t}^{-\beta} u^{\prime}(t)+\frac{1}{2}{ }_{t} D_{b}^{-\beta} u^{\prime}(t)\right)+\lambda u(t)=0, \quad a<t<b \\
u(a)=u(b)=0
\end{array}\right.
$$


depending on the parameter $\lambda \in \mathbb{R}$. If problem (3.6) has a nontrivial weak solution in $E^{\alpha}$, then $\lambda$ is an eigenvalue of problem (3.6) and $u_{\lambda}$ is a eigenvalue function associated to $\lambda$. From [13, Theorem 3.1], we know that

$$
\lambda_{1}=\inf _{u \in E^{\alpha} \backslash\{0\}} \frac{-\int_{a}^{b} D_{t}^{\alpha} u(t)_{t} D_{b}^{\alpha} u(t) d t}{\int_{a}^{b} u^{2}(t) d t} .
$$

In the following conclusion, we provide lower bounds for $\lambda_{1}$.

Corollary 3.5. Let $\lambda_{1}$ be the first eigenvalue of problem (3.6) given by (3.7). Then, we have

$$
\lambda_{1} \geqslant\left(\frac{2(b-a)^{\left(\alpha-\frac{1}{2}\right)}}{\Gamma(\alpha)(2 \alpha-1)^{\frac{1}{2}}} \frac{1}{|\cos (\pi \alpha)|^{\frac{1}{2}}}\right)^{-2} .
$$

Proof. It follows from Theorem 1.1 by taking $\omega=\lambda_{1}$.

\section{Acknowledgment}

Yeong-Cheng Liou was supported in part by the grand form Kaohsiung Medical University Research Foundation (KMU-Q106005) and Taiwan-Russian joint grant MOST 106-2923-E-039-001-MY3.

\section{References}

[1] R. C. Brown, D. B. Hinton, Lyapunov inequalities and their applications, Survey on classical inequalities, Math. Appl., Kluwer Acad. Publ., Dordrecht, 517 (2000), 1-25. 1

[2] A. Cañada, J. A. Montero, S. Villegas, Lyapunov inequalities for partial differential equations, J. Funct. Anal., 237 (2006), 176-193. 1

[3] A. Cañada, S. Villegas, Lyapunov inequalities for Neumann boundary conditions at higher eigenvalues, J. Eur. Math. Soc. (JEMS), 12 (2010), 163-178. 1

[4] P. L. de Nápoli, J. P. Pinasco, A Lyapunov inequality for monotone quasilinear operators, Differential Integral Equations, 18 (2005), 1193-1120. 1

[5] Á. Elbert, A half-linear second order differential equation, Qualitative theory of differential equations, Vol. I, II, Szeged, (1979), Colloq. Math. Soc. János Bolyai, North-Holland, Amsterdam-New York, 30 (1981), 153-180. 1

[6] R. A. C. Ferreira, A Lyapunov-type inequality for a fractional boundary value problem, Fract. Calc. Appl. Anal., 16 (2013), 978-984. 1

[7] R. A. C. Ferreira, On a Lyapunov-type inequality and the zeros of a certain Mittag-Leffler function, J. Math. Anal. Appl., 412 (2014), 1058-1063. 1

[8] F. Jiao, Y. Zhou, Existence of solutions for a class of fractional boundary value problems via critical point theory, Comput. Math. Appl., 62 (2011), 1181-1199. 3, 3.1, 3, 3.4

[9] M. Jleli, M. Kirane, B. Samet, Lyapunov-type inequalities for fractional partial differential equations, Appl. Math. Lett., 66 (2017), 30-39. 1

[10] M. Jleli, B. Samet, Lyapunov-type inequalities for a fractional differential equation with mixed boundary conditions, Math. Inequal. Appl., 18 (2015), 443-451 . 1

[11] M. Jleli, B. Samet, Lyapunov-type inequalities for fractional boundary-value problems, Electron. J. Differential Equations, 2015 (2015), 11 pages. 1

[12] A. A. Kilbas, H. M. Srivastava, J. J. Trujillo, Theory and applications of fractional differential equations, North-Holland Mathematics Studies, Elsevier Science B.V., Amsterdam, (2006). 2

[13] Y.-N. Li, H.-R. Sun, Q.-G. Zhang, Existence of solutions to fractional boundary-value problems with a parameter, Electron. J. Differential Equations, 2013 (2013), 12 pages. 3

[14] D. O’Regan, B. Samet, Lyapunov-type inequalities for a class of fractional differential equations, J. Inequal. Appl., 2015 (2015), 10 pages. 1

[15] J. P. Pinasco, Lyapunov-type inequalities, With applications to eigenvalue problems, SpringerBriefs in Mathematics. Springer, New York, (2013). 1

[16] J. Rong, C.-Z. Bai, Lyapunov-type inequality for a fractional differential equation with fractional boundary conditions, Adv. Difference Equ., 2015 (2015), 10 pages. 1

[17] A. Tiryaki, Recent developments of Lyapunov-type inequalities, Adv. Dyn. Syst. Appl., 5 (2010), 231-248. 1 